\title{
The level of effort required for behaviour as a moderator of the attitude-behaviour relation*
}

\author{
RICHARD P. BAGOZZI AND YOUJAE YI \\ The University of Michigan, U.S.A. \\ and JOHANN BAUMGARTNER \\ Pennsylvania State University, U.S.A.
}

\section{Abstract}

It was hypothesized that the manner in which attitudes influence behaviour is moderated by the level of effort required to perform a behaviour. The effort needed was manipulated in a field experiment by varying the difficulty of getting access to the attitude object. When the behaviour required substantial effort, the mediating role of intentions was strong, and attitudes had only indirect effects on behaviour, consistent with the theory of reasoned action. When the behaviour required little effort, however, attitudes had a significant direct effect on behaviour, and the mediating role of intentions was reduced. The theoretical and practical implications of these findings are discussed.

\section{INTRODUCTION}

A substantial body of research has investigated the attitude-behaviour relation with an aim toward answering the question, 'How do attitudes influence behaviour?' Research on this issue has relied heavily upon the theory of reasoned action (Ajzen and Fishbein, 1980; Echabe, Rovira and Garate, 1988). This theory maintains that attitudes influence behaviour only indirectly through their impact on intentions. It is hypothesized and in fact has been frequently found that intentions fully mediate the effects of attitudes on behaviour (e.g. Fredricks and Dossett, 1983).

However, some researchers have discovered that attitudes can influence behaviour directly (e.g. Bentler and Speckart, 1979; Manstead, Proffitt and Smart, 1983). For example, Bentler and Speckart (1981) found substantial and statistically significant effects of attitudes on behaviour, controlling for behavioural intentions. Some studies even found that attitudes are better predictors of behaviour than are behavioural intentions (e.g. Albrecht and Carpenter, 1976; Bentler and Speckart, 1979, 1981).

\footnotetext{
* The authors wish to thank the Editor and an anonymous reviewer for their comments on an earlier
} version of this manuscript. 
In sum, these studies suggest that intentions may not completely mediate the effects of attitudes on behaviour, and may even be less important as a determinant of behaviour.

Understanding the role of intentions in the attitude-behaviour relation is important for several reasons. From a theoretical viewpoint, knowing when attitudes function indirectly by affecting behaviour through intentions or directly by by-passing intentions can aid in the explanation of the etiology of behaviour. This will provide a better understanding of mechanisms underlying the attitude-behaviour relation.

From a more pragmatic standpoint, understanding the role of intentions can guide persuasion strategies. If intentions fully mediate the effects of attitudes on behaviour as a necessary link, communication strategies must take into account the nature and timing of intention formation, as well as attitude formation, if they are to lead to behaviour change. On the other hand, if attitudes have direct effects unmediated by intentions, then communication strategies may be fruitfully aimed at inducing affect (e.g. via emotional appeals) as a route to behaviour change. In such cases, the retrieval of an emotionally charged attitude or the creation of a new affective response may directly produce changes in behaviour.

Despite the theoretical and practical importance of understanding the role of intentions in the attitude-behaviour relation, existing findings about the issue are mixed and inconclusive, as reviewed earlier. The present study attempts to shed new light on this issue by taking a different perspective. Rather than asking whether or not intentions mediate the attitude-behaviour relation, the following question is asked: When do intentions mediate the attitude-behaviour relation? The purpose of the present study is therefore to identify certain conditions under which intentions may or may not mediate the effects of attitudes on behaviour.

\section{EFFORT REQUIRED FOR BEHAVIOUR}

It is hypothesized in this article that the level of effort required to perform a behaviour determines the role of intentions in mediating the effects of attitudes on behaviour. Specifically, it is hypothesized that the mediating role of intentions will be greater when the level of effort needed for behaviour is high than when it is low. It is also hypothesized that attitudes will have stronger direct effects on behaviour when performance of the behaviour requires low rather than high effort. The level of effort necessary to perform a behaviour refers herein to the degree of difficulty in executing the behaviour and is a function of impediments to performance of the behaviour.

The role of effort needed for the performance of a behaviour can be interpreted in the following way. First, a new attitude forms or an existing attitude is retrieved from memory. This implies that the desire to approach the attitude object or perform the target act will be a function of the strength of one's attitude. When the act requires little effort or when impediments to performance of the act are absent, attitude may directly lead to behaviour. In such cases, attitudes can stimulate an action with little thought. On the other hand, when the act needs much effort or when impediments to performance of the act are present, a certain amount of planning and deliberation is required. Attitudes here must be strong enough to influence intentions to act if it is to lead to behaviour. 
To date, little research has been done linking the amount of effort needed for the performance of a behaviour to the role of intentions. However, an examination of the meaning of intentions suggests that their mediating role may differ depending upon the amount of effort that performing a behaviour requires. For example, intention has been defined as 'the degree to which a person has formulated conscious plans to perform or not perform some specified future behaviour' (Warshaw and Davis, 1985)'. Webster's New Collegiate Dictionary (1980, p. 596) defines intention as, 'a determination to act in a certain way' and to intend as 'to have in mind as a purpose or goal'. Synonyms of intention include 'deliberateness, calculation, willingness, determination' (Roget's Thesaurus, 1966, p. 361). The shared meaning throughout these synonyms of intention seems to be 'behavioural commitment to perform a behaviour' or 'deliberate planning to attain a goal'.

The above meaning of intentions suggests circumstances under which intentions might be relevant in the attitude-behaviour relation. Since intentions can be conceptualized as deliberate planning or commitment, they should be important predictors of behaviours in situations where performance of behaviours requires considerable effort. Performance of some behaviours (e.g. exercising every day) is relatively difficult and requires substantial planning and commitment. For example, one may like the idea of exercising daily, but unless one is willing to make conscious and deliberate plans, performance of the behaviour is unlikely. One's intentions toward the behaviour become necessary causes of the behaviour. Other behaviours (e.g. reading a newspaper) may be so easy to perform as to require little deliberation and commitment. Intentions here may not be necessary, and attitudes directly lead to behaviour ${ }^{2}$. In sum, intentions are likely to become a less important predictor of a behaviour as the level of effort required to perform the behaviour decreases.

Suppose we are interested in explaining purchases of beer. The purchase of beer may take little effort in certain cases, as in most areas of the United States where it is easily available in a variety of outlets such as drug, liquor, grocery, supermarket, and other stores. In such cases, favourable attitudes toward beer may be sufficient to cause the purchase behaviour with little or no planning and effort expended. People can simply take beer from the shelf during normal grocery shopping or other shopping trips even when they do not have previous plans or intention per se. On the other hand, buying beer may require substantial effort if it is available only at state-run liquor stores during certain hours of the day, as is the practice in certain areas of the United States. In such situations, rather involved decision processes must be undertaken each time one desires to purchase beer (e.g. plans must be made to stop by the liquor store during a particular time of the day). Although one has a favourable attitude toward beer, one will not buy it unless one exerts considerable effort to do so. As a consequence, more involved processes are required if one is to purchase beer. The hypotheses are thus: (1) intentions will play a smaller role, and (2) attitudes will play a greater role, in determining behaviour when the level of effort needed to perform the behaviour is low than when it is high.

\footnotetext{
' Warshaw and Davis (1985) note that behavioural intentions should be distinguished from behavioural expectations, which have been defined as 'an individual's estimation of the likelihood that he or she actually will perform some specified future behaviour'.

${ }^{2}$ It should be noted that the same behaviour can be either easy or difficult to perform, depending upon the situation. For some people, reading a newspaper is a regular activity driven more by habit and stored attitudes than new volitions.
} 


\section{METHOD}

\section{Subjects}

Subjects were 166 students enrolled in four sections of an introductory business course. Two questionnaires, separated by one week, were administered during class time in the winter term. Ninety-two per cent (152 students) of the original pool of subjects, who had been asked to participate in a study of business curriculum, were present in both waves of data collection.

\section{Procedure}

The target behaviour was readership of a written follow-up of one case analysis that students discussed in the previous class. This behaviour was chosen because it is under volitional control, satisfying the boundary condition of the theory of reasoned action (Fishbein and Ajzen, 1975). Students were required to analyse a number of cases for class discussion throughout the term. Some cases had follow-ups that illustrated the actual decisions made by firms and the results of the decision. A portion of these follow-ups was made available to the students after class discussion.

Pilot interviews with students similar to those students in the main investigation from the previous term showed that they tended to read the case follow-ups voluntarily. Since the case follow-ups were stipulated as optional readings by instructors and the course syllabus clearly stated that case materials would not be included in examinations, the behaviour has a decidedly voluntary character to it. Students read the follow-ups because they were intrinsically interested in the results and actual decisions taken by firms in the case and not because they felt that they would be directly rewarded for reading or punished for failing to read the follow-up.

The level of effort required to read the case follow-up was manipulated by the instructors. The manipulation was designed to create conditions such that getting access to the attitude object to perform the target behaviour required different levels of effort from the subjects. At the same time, the manipulation had to be realistic in view of the nature of the present study (a field experiment). Pretests indicated that this could be achieved by employing two different, but often-used, methods of making the supplementary readings available to the students. In the high effort condition, students were told during class time that five copies of the follow-up to the case were put on reserve in the library. In this condition, students had to go to the library and fill out a form for checking out the material in order to read the follow-up. Since the number of the copies was limited (five), it was also likely that students might have to wait when all copies were checked out. These factors (i.e. location in the library, five copies) would make it necessary for the target behaviour to demand relatively substantial effort. On the other hand, in the low effort condition, a copy of the follow-up was distributed to each student during the class by the instructor. Two sections of classes were randomly assigned to either high or low effort conditions, respectively. In this way, 79 subjects were in the low effort condition, whereas 73 were in the high effort condition.

The study was begun three weeks before mid-term exams so as to avoid both the beginning of the term and exam periods which are typically hectic. An interviewer came to the classrooms and administered the first questionnaire. It was made clear 
to subjects that the questionnaire was part of a study conducted by the school independently of the instructors. Although the questionnaire was self-administered, the interviewer remained in the classroom in order to answer any questions from subjects.

On the first page of the questionnaire, a short description of the study was given as follows: 'We are interested in updating and developing the curriculum for courses. For that purpose, we would like to know your opinions about cases for the course, XXX. Your responses will be kept strictly anonymous. No one will know who the respondents are'. This page also contained questions pertaining to subjects' attitudes toward cases and case follow-ups in general. These latter measures were included so that they could be used to check the effectiveness of the randomization procedure. The next page contained the measures of attitudes, subjective norms, and intentions toward the target behaviour (reading the follow-up of a case during the upcoming week). These items are described later under Measures.

Subjects were asked to write the last four digits of their student numbers (a 10-13 digit code) at the end of the questionnaire. This was done to match up subjects across the two waves and to reinforce assurance of anonymity given on the questionnaire and verbally by the person administrating the questionnaire. It should also be noted that the administrator of the questionnaire was not an instructor of any of the students and was blind to the true purpose of the study. These steps were taken in order to reduce any demand characteristics induced by the study.

The second questionnaire was administered to the subjects one week after the first. An interval of one week was used so that the time lag between the measurement of attitudes/intentions and behaviour would not be excessive (Davidson and Jaccard, 1979). Subjects were asked about their performance of the target behaviour (reading of the follow-up to the case) during the previous week. This questionnaire also included measures of the perceived level of effort required to read the follow-up, which were used for manipulation checks. These measures are described later under Measures.

The study concluded with a short debriefing of the subjects. The debriefing explained the general purpose of the study and then detailed the experimental design and hypotheses. Respondents were told that they could see the results of the study by contacting the authors and they were again thanked for their cooperation.

\section{Measures}

Multiple indicators (measures) were used to assess the reliability of measurements for each variable and to remove measurement error as a by-product of analysis of latent variable models described below (except for subjective norms). Attitudes (Att) toward the target behaviour were measured by asking subjects to express their attitude on 7-point scales. Subjects were asked, 'My attitude toward reading a followup of a case during the upcoming week can be best described as ...' Three bipolar adjectives (Att1, Att2, and Att3) describing the subject's feelings were used: good/bad, pleasant/unpleasant, and favourable/unfavourable. The subjective norm (SN) variable was measured with the standard item (Fishbein and Ajzen, 1975): 'Most people who are important to me think I should/should not read a follow-up of a case during the upcoming week'. A 7-point $(-3$ to +3$)$ scale was used to record responses.

Intentions (BI) were measured by asking subjects to express their intentions and 
plans to read a follow-up of a case during the upcoming week. One measure (BI1) was a 7-point 'definitely intend to/definitely intend not to' item. Another measure of intentions (BI2) was a 7-point 'definitely plan to/definitely plan not to' item. These measures are consistent with standard measures of intentions and should be unconfounded with behavioural expectations ( $c f$. Warshaw and Davis, 1985).

Behaviours (B) were assessed by three indicators. One item (B1) asked subjects approximately how many minutes they spent on reading the follow-up. A second indicator of behaviour (B2), concerning the depth of the subjects' reading, asked, 'How much did you read of the follow-up to the case?' This was measured by a 7-point scale with $0=$ 'read none of it', $6=$ 'read it carefully more than once', and 2-5 = 'read a part', 'skimmed it', 'read it casually' and 'read it carefully', respectively. The final item (B3) was an objective indicator and assessed the degree of subjects' knowledge about the content of the follow-up to the case. Subjects who indicated having read the follow-up were asked to answer 10 questions about specific content in the follow-up in the form of a multiple choice recognition test. An example question included, 'Which advertising media did the firm use?' The number of correct answers was used as the final behavioural measure (B3), with $0=$ minimum and $10=\operatorname{maxi}$ mum. Those subjects who indicated they had not read the follow-up were assigned a score of $\mathbf{0}$ for this measure. It should be noted that two measures of behaviour (i.e. B1 and B3) were ratio-scaled. This is in contrast with many previous studies which used behaviour measures expressed as dichotomous responses, resulting in lost information and non-normal distributional properties.

Subjects' attitudes toward cases and follow-ups in general were measured in order to assess the initial equivalence between the two treatment groups. Subjects' attitudes toward cases in general (Attc) were assessed by a Likert-type scale of four items at the beginning of the first questionnaire. For example, one item was, 'Cases are interesting and stimulating'. Subjects indicated their opinions for each item on a 5-point 'strongly disagree' to 'strongly agree' scale. Immediately after these questions, attitudes toward case follow-ups (Attf) were similarly assessed with four items (e.g. 'I am interested in follow-ups, if any').

To assess whether the manipulation was successful, the level of effort required to perform the target behaviour was measured with two items in the second questionnaire. One item (Effl) asked subjects to indicate the difficulty of getting access to the follow-up. A 7-point scale was used with the end points of 'extremely available' (1) and 'extremely unavailable' (7). Another item (Eff2) asked the subjects how inconvenient they perceived it to obtain the case follow-up for reading. Subjects answered on a 7-point scale ranging from 'extremely convenient' (1) to 'extremely inconvenient' (7).

\section{Analysis}

A structural equation model with latent variables was specified for the relations among attitudes, subjective norms, intentions, and behaviour, as shown in Figure 1. In accordance with the theory of reasoned action, attitudes and subjective norms are hypothesized to be determinants of behavioural intentions, which in turn are immediate precursors to behaviour. However, as a hypothesis, a direct path from attitudes to behaviour was also included in the model in accordance with Bentler and Speckart's $(1979,1981)$ view. The direct path from subjective norms to behaviour 


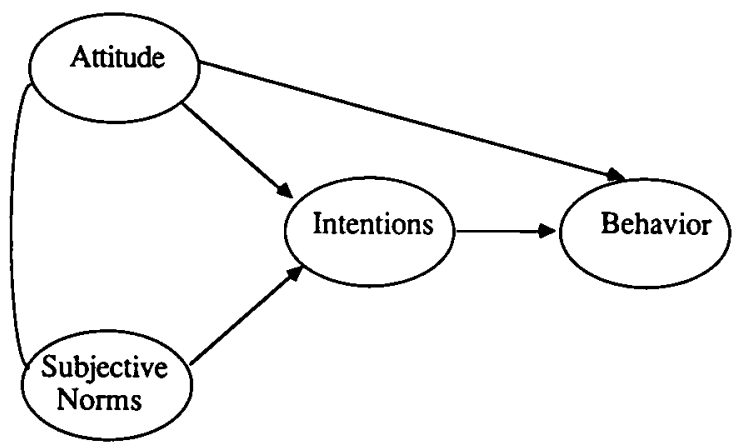

Figure 1. Causal model used in the study (only latent variables are shown for simplicity)

was not specified, since such a path was neither hypothesized nor found by previous researchers (Ajzen and Fishbein, 1980; Bentler and Speckart, 1979, 1981) ${ }^{3}$.

The model in Figure 1 was estimated with the LISREL VI program (Joreskog and Sorbom, 1984). Multiple observed indicators of latent constructs were used to infer relationships among the variables. The maximum likelihood function was used to estimate parameters and test hypotheses. Multiple group analyses were conducted in order to compare the key parameters across the two groups. In such analyses, the two groups were analysed simultaneously in order to test the equality of the key parameters across groups. The maximum likelihood fitting function for the simultaneous analysis is the weighted average of the fitting functions for each group in the analysis (e.g. Joreskog, 1971; Joreskog and Sorbom, 1984).

Tests of invariance hypotheses were conducted in two ways. First, a simultaneous analysis was run with the desired invariance constraints specified for the two groups. A significant chi-square test points to a rejection of the hypothesis of invariance, while a nonsignificant value will lead to a failure to reject invariance. Second, chisquare difference tests were used by comparing the model with invariance constraints to the model allowing parameters of interest to be free and unconstrained. Since these models were nested, the difference in chi-squares between the models should be distributed as chi-square with degrees of freedom equal to the difference between the number of free parameters (Joreskog and Sorbom, 1984). A chi-square difference corresponding to a probability level of less than 0.05 was used as a criterion to reject the null hypothesis that the given parameters are equal across the two groups. All tests were performed on the covariance matrix, since they involve multiple group analyses (Joreskog and Sorbom, 1984).

\section{RESULTS}

\section{Randomization and manipulation checks}

Although the student sections were randomly assigned to the experimental treatment, it was checked whether randomization was achieved by comparing the subjects' general attitudes toward cases and follow-ups. Results showed that subjects'attitudes

${ }^{3}$ A model with the direct path from subjective norms to behaviour was also estimated in this study, but the path was not significant, as anticipated. 
toward cases in general (Attc) did not differ between the low $(M=16.0)$ and high $(M=15.9)$ conditions $(t=0.22, p>0.8)$. It was also found that attitudes toward case follow-ups (Attf) were not different between the low (15.2) and high (15.3) conditions $(t=0.07, p>0.9)$. These checks give a rough indication that the two groups were initially equivalent.

The manipulation of the effort level required to perform the target behaviour (i.e. read the case follow-up) was checked with two items in the second questionnaire (Eff1 and Eff2). Results on the first item (Eff1) showed that subjects perceived it to be easier to get the case follow-up in the low $(M=1.8)$ than high $(M=4.5)$ effort condition $(t=13.3, p<0.001)$. The results on the second item (Eff2) showed that it was more convenient to get the case follow-up in the low $(M=2.1)$ than high $(M=4.8)$ effort condition $(t=9.9, p<0.001)$. When the sum of the two items was used as an index of the level of effort needed $(E f f=E f f 1+E f f 2)$, the results ( $M=3.9$ versus $9.3, t=14.2, p<0.001)$ suggested the same conclusion; performing the target behaviour was less effortful in the low than high effort group.

\section{Reliabilities and overall model fit}

We computed the reliabilities of individual items, composite reliabilities, and average variance extracted (AVE) for all the measures, except for subjective norm which was measured with a single item (Werts, Linn and Joreskog, 1974). Table 1 summarizes the results. Most of the individual item reliabilities are high. All composite reliabilities are very high, with the average being 0.92 . Further, all the AVE measures are greater than or equal to 0.8 , a level much above the 0.5 rule-of-thumb. The mean AVE was 0.83 , suggesting that more than 80 per cent of the variance in the constructs was accounted for by the measures. Overall, the measures of key constructs were found to be quite reliable.

Table 2 summarizes the means and standard deviations of the key variables in the low and high effort groups. The sum of the items for each key variable was used as an overall score of the variable in this table (e.g. Attitude $=$ Attl + Att2 + Att3). The mean attitude scores were 14.48 and 14.16 for the low effort and high effort groups, respectively. The mean scores for subjective norm and intentions were 1.13 versus 1.05 and 9.66 versus 7.96 for both groups. As expected, the behaviour measure showed a higher mean in the low effort group than in the high effort group ( 9.84 versus 1.03$)$.

Table 3 shows overall goodness-of-fit measures and estimates of key parameters of the model in Figure 1 from a simultaneous analysis of the low and high effort groups. The overall model was assessed by checking whether the specified model provides a satisfactory fit to the observed data. The fit of the model was assessed by two global measures: chi-square tests and the goodness-of-fit (GFI) index.

First, the results for the model were examined with the chi-square test. The chisquare test indicates the degree of a model fit to the observed data. A nonsignificant chi-square value supports the hypothesis that the model provides a plausible representation of the underlying processes. For this simultaneous group analysis, the chisquare value was $\chi^{2}(46)=39.4, p=0.75$. By the usual rule-of-thumb regarding the $p$-value $(p \geq 0.05)$, the model fit was satisfactory. Separate analysis of each group also showed that the model fit is satisfactory: $\chi^{2}(23)=24.1, p=0.40 ; \chi^{2}(23)=15.3$, $p=0.89$, for high and low effort groups respectively. Second, the goodness-of-fit 
Table 1. Reliabilities of the measures

\begin{tabular}{llcc}
\hline Measures & $\begin{array}{c}\text { Individual } \\
\text { item } \\
\text { reliability }\end{array}$ & $\begin{array}{c}\text { Composite } \\
\text { reliability }\end{array}$ & $\begin{array}{c}\text { Average } \\
\text { variance } \\
\text { extracted }\end{array}$ \\
\hline Attitude & $0.83(0.71)$ & $0.92 *(0.93 \dagger)$ & $0.80(0.82)$ \\
Att1 & $0.68(0.79)$ & & \\
Att2 & $0.90(0.95)$ & $0.95(0.94)$ & $0.90(0.88)$ \\
Att3 & $0.98(0.99)$ & & \\
Intention & $0.81(0.78)$ & $0.94(0.82)$ & \\
BI1 & & & \\
BI2 & $0.61(0.45)$ & & \\
Behaviour & $0.99(0.97)$ & & \\
B1 & $0.90(0.87)$ & & \\
B2 & B3 & & \\
\hline
\end{tabular}

* Reliability estimates for the high effort group $(n=73)$.

$\dagger$ Reliability estimates for the low effort group $(n=79)$.

Table 2. Means and standard deviations of key variables

\begin{tabular}{lcccc}
\hline & \multicolumn{2}{c}{ Low effort group } & \multicolumn{2}{c}{ High effort group } \\
$(n=73)$ & & \multicolumn{2}{c}{$(n=79)$} & \\
Variables & Mean & s.d. & Mean & s.d. \\
\hline Attitude* & 14.48 & 4.51 & 14.16 & 4.09 \\
Subjective norm $\dagger$ & 1.13 & 0.81 & 1.05 & 0.83 \\
Intention $\ddagger$ & 9.66 & 2.76 & 7.96 & 2.85 \\
Behaviour§ & 9.84 & 6.95 & 1.03 & 3.03 \\
\hline
\end{tabular}

*Attitude $=$ Att $1+$ Att $2+$ Att 3 .

$\dagger$ Subjective norm $=\mathrm{SN}$.

$\ddagger$ Intention $=\mathrm{BI} 1+\mathrm{BI}$.

$\S$ Behaviour $=\mathbf{B} 1+\mathbf{B} 2+\mathbf{B} 3$.

Table 3. Key paths for low and high effort groups

\begin{tabular}{lrrrrrr}
\hline & \multicolumn{3}{c}{ Low effort group } & \multicolumn{3}{c}{ High effort group } \\
Path & Unstd. & \multicolumn{1}{c}{ Std. $\dagger$} & $t$-value & Unstd. ${ }^{*}$ & Std. $\dagger$ & $t$-value \\
\hline $\mathrm{A} \rightarrow$ BI & 0.36 & 0.30 & 2.6 & 0.42 & 0.38 & 3.1 \\
SN $\rightarrow$ BI & -0.04 & -0.04 & 0.3 & -0.15 & -0.15 & 1.3 \\
BI $\rightarrow$ B & 0.04 & 0.07 & 0.6 & 0.31 & 0.40 & 3.1 \\
A $\rightarrow$ B & 0.28 & 0.35 & 2.8 & 0.06 & 0.07 & 0.6 \\
\hline
\end{tabular}

* Unstandardized estimates.

† Standardized estimates.

Model fit results are $\chi^{2}(46)=39.4, p=0.75$.

(GFI) index was 0.96 , indicating that a substantial amount of the variances and covariances were jointly accounted for by the hypothesized model. Finally, all normalized residuals were less than 2.0 in magnitude, further suggesting that no model specification errors were present. 
Table 4. Tests of invariance for key paths

\begin{tabular}{lccc}
\hline Path & \multicolumn{2}{c}{ Fit of the model with the path } & Fixed to be equal \\
Free & $\begin{array}{c}\text { Test of invariance } \\
\chi^{2}{ }_{\mathrm{d}} \text {-test }\end{array}$ \\
\hline $\mathrm{A} \rightarrow \mathrm{BI}$ & $\chi^{2}(46)=39.4$ & $\chi^{2}(47)=39.6$ & $\chi^{2}{ }_{\mathrm{d}}(1)=0.2$ \\
& $p=0.75$ & $p=0.77$ & $p=0.70$ \\
$\mathrm{SN} \rightarrow \mathrm{BI}$ & $\chi^{2}(46)=39.4$ & $\chi^{2}(47)=39.7$ & $\chi^{2}(1)=0.3$ \\
& $p=0.75$ & $p=0.77$ & $p=0.60$ \\
$\mathrm{BI} \rightarrow \mathrm{B}$ & $\chi^{2}(46)=39.4$ & $\chi^{2}(47)=701.2$ & $\chi^{2}{ }_{\mathrm{d}}(1)=661.8$ \\
& $p=0.75$ & $p=0.00$ & $p=0.00$ \\
$\mathrm{~A} \rightarrow \mathrm{B}$ & $\chi^{2}(46)=39.4$ & $\chi^{2}(47)=563.8$ & $\chi^{2}{ }_{\mathrm{d}}(1)=524.4$ \\
& $p=0.75$ & $p=0.00$ & $p=0.00$ \\
\hline
\end{tabular}

\section{Tests of hypotheses}

It was hypothesized that effort needed to perform the behaviour would moderate the role of intentions in mediating the attitude-behaviour relation. This hypothesis was examined by comparing the parameter estimates between the low and high effort groups. Subjects who had participated in the study were assigned to low and high effort conditions in the manner described earlier. Table 3 provides the regression weights for the key paths.

First, the direct path from attitudes to behaviour was examined. The direct effects of attitudes, unmediated by intentions, were significant for the low effort group $\left(\gamma^{\mathrm{L}}=0.28, t=2.8\right)$. However, for the high effort group the direct effect was statistically insignificant $\left(\gamma^{\mathrm{H}}=0.06, t=0.6\right)$. Second, the path from intentions to behaviour was examined. For the low effort group, intentions did not have significant effects on behaviour $\left(\beta^{\mathrm{L}}=0.04, t=0.6\right)$. But for the high effort group, intentions did have significant effects on behaviour $\left(\beta^{\mathrm{H}}=0.31, t=3.1\right)$.

Further, the effects of attitudes and subjective norms on intentions were examined. In each group, attitudes had significant effects on intentions $\left(\gamma^{\mathrm{L}}=0.36, t=2.6\right.$; $\gamma^{\mathrm{H}}=0.42, t=3.1$, respectively). But subjective norms had no significant effects on intentions in either group $\left(\gamma^{\mathrm{L}}=-0.04, t=0.3 ; \gamma^{\mathrm{H}}=-0.15, t=1.3\right.$, respectively).

As hypothesized, the mediating role of intentions was contingent on the level of effort required to perform the behaviour. The effects of attitudes were fully mediated by intentions under high effort conditions. On the other hand, under low effort conditions the mediating role of intentions was eliminated, and attitudes had direct, unmediated effects on behaviour.

Although the above analyses gave support for the hypotheses of this study, a more rigorous test was conducted through multiple group analyses. For each one of the four key paths, the difference in parameter values across groups was tested for statistical significance by invariance tests with a simultaneous group analysis. Table 4 summarizes the results from the tests of invariance across the two groups.

First, the invariance of the effect of intentions on behaviour was tested. For this purpose, the path from intentions to behaviour was constrained to be equal for the two groups. The multiple group analysis yielded the following results: $\chi^{2}(47)=701.2, p=0.00$. Tests of invariant effects were performed with a chi-square difference test by comparing the finding for the constrained model with a model allowing all parameters of interest (including a path from intentions to behaviour) 
to be free and unconstrained. See the final column in Table 4 for these chi-square difference tests. The unconstrained model with all parameters free yielded the following chi-square statistics: $\chi^{2}(46)=39.4, p=0.75$ (see column 2 in Table 4). Therefore, the chi-square difference is 661.8 with one degree of freedom, which is significant at the 0.001 level. This gives strong evidence that the effects of intentions on behaviour varied with the level of effort needed for performance of a behaviour. Overall, intentions had stronger effects on behaviour when a high rather than low level of effort is needed $\left(\beta^{\mathrm{L}}=0.04\right.$ versus $\left.\beta^{\mathrm{H}}=0.31\right)$.

The invariance of the direct effects of attitudes on behaviour was also tested. The multiple group analysis assuming invariant direct effects provided the following goodness-of-fit measures: $\chi^{2}(47)=563.8, p=0.00$. When this finding was compared with that for the unconstrained model, the chi-square difference $\left(\chi^{2}{ }_{d}\right)$ was 524.4 with one as the difference in degrees of freedom. The null hypothesis of invariant direct effects was thus rejected at the 0.001 level, and there is strong evidence suggesting that direct effects are different between the groups. Combined with the findings in Table 3, the results suggest that attitudes had stronger direct effects, unmediated by intentions, on behaviour when behaviour requires low than high effort $\left(\gamma^{\mathrm{L}}=0.28\right.$ versus $\gamma^{\mathrm{H}}=0.06$ )

The effects of attitudes on intentions were also tested for their invariance across the two groups. The constrained model assuming equality gave: $\chi^{2}(47)=39.6$, $p=0.77$. The chi-square difference is: $\chi_{\mathrm{d}}^{2}(1)=0.2, p=0.7$. Therefore, one cannot reject the null hypothesis of invariance across the two groups. Attitudes influenced intentions equally well in both groups. Finally, the invariance of the effects of subjective norm on intentions was tested similarly. The multiple group analysis assuming this invariance yielded the following results: $\chi^{2}(47)=39.7, p=0.6$. The chi-square difference is: $\chi^{2}{ }_{d}(1)=0.3, p=0.6$. Hence, consistent with predictions, the effects of attitude and subjective norms on intentions did not differ between low and high effort conditions.

\section{DISCUSSION}

Liska (1984) has recently suggested that attitude theorists have tended to view behaviour as strictly volitional or strictly nonvolitional, perhaps creating a dichotomy that really does not exist for at least some, if not at all, actions. The theory of reasoned action is clear in its applicability to volitional behaviours, and a vast body of empirical findings support this model (e.g. Ajzen and Fishbein, 1980; Fishbein and Ajzen, 1975). However, a number of recent studies seem to challenge the model by finding evidence for a direct effect of attitudes on behaviour, controlling for intentions (e.g. Bentler and Speckart, 1979, 1981; Manstead et al., 1983; Zuckerman and Reis, 1978). The question remains: Why do attitudes sometimes have an indirect effect (through intentions) or a direct effect on behaviour?

Following Zanna and Fazio (1982), we have chosen to focus on the 'when' question and study the conditions moderating the effects of attitudes on behaviour, instead of merely addressing the 'is' question, as done so far in the literature, which asks only whether attitudes can or do influence behaviour directly and indirectly. That is, rather than asking, 'Is intention a necessary mediator of the attitude-behaviour 
relation?', we have attempted to answer the question, 'Under what conditions might attitudes indirectly (through intentions) or directly affect behaviour?'

The results of this investigation support the hypothesis that the level of effort needed to perform a behaviour moderates the role of intentions in the attitudebehaviour relation. When the level of effort required for behaviour was high, intentions fully mediated the effects of attitudes on behaviour, and thus attitudes had no direct effects on behaviour. This finding is in accordance with the classic sequence hypothesized by Fishbein and Ajzen (1975) and Triandis (1977). On the other hand, when behaviour required little effort, intentions did not completely mediate the effects of attitudes, and attitudes had direct effects on behaviour. This is consistent with recent findings (e.g. Bentler and Speckart, 1979, 1981). In sum, the mediating role of intentions varied along a continuum from effortful to effortless behaviours.

Findings of this study provide useful insights into the process of behavioural determination and attitude-behaviour relations (e.g. Fazio, 1986). Behaviours requiring much effort are determined largely by deliberate processes in which individuals participate in conscious thought processes to arrive at behavioural intentions, a point of view consistent with the theory of reasoned action. But behaviours requiring little effort are guided by less deliberate processes in which behavioural decisions are stimulated directly by attitudes, a finding consistent with recent research (e.g. Bentler and Speckart, 1981). The present investigation suggests one way in which the two streams of research can be integrated. That is, direct versus indirect effects of attitudes depend upon the effort needed for performance of the act. In the present study, the target behaviour of reading the case follow-up clearly involves volitional control. In this regard, the findings are interesting since attitude affect behaviour under volitional control differently depending upon the effortfulness of behaviour.

The results of this study show that attitudes have direct or indirect effects (via intentions) on behaviour. Nevertheless, there is considerable evidence in dissonance and self-perception research that behaviour affects attitudes (e.g. Bem, 1972; Festinger, 1957). In this study, however, since attitudes were measured before the target act and subjects had no previous experience with the target act, the reversal of the attitude-behaviour link is not likely to pose a threat to our findings (that is, the paths from prior attitudes to behaviour). If attitudes had been measured again after the target behaviour, the link from behaviour to subsequent attitudes could be estimated using a cross-lagged panel analysis (e.g. Bentler and Speckart, 1981; Kahle and Berman, 1979). Furthermore, the dissonance theory would predict that a deliberate choice of an act requiring high effort would increase one's liking for the act (e.g. due to effort justification) $)^{4}$. An implication is that attitude changes will be greater for those who performed the target behaviour under high than low effort conditions. This is a very interesting hypothesis, which should be tested in future research.

The path from subjective norms to intentions was insignificant in both the low and high effort groups in this study. A possible explanation for this lies in the nature of the target behaviour: reading a follow-up to a case discussed in class. We suspect that little normative pressure from instructors, peers, or others was felt by subjects. Subjects were told that the follow-ups were optional readings, would not be discussed in class, and would not serve as content in examinations or homework assignments.

\footnotetext{
${ }^{4}$ We thank the Editor for suggesting this interesting idea.
} 
This tends to weaken any felt expectations of the instructors or others. Indeed, the mean response of the subjective norm measures in the two groups $(M=1.12$ versus 1.05) indicates that low levels of overall social influence existed with respect to reading case follow-ups in this study (see Table 2).

On the other hand, differential (e.g. significant or insignificant) effects of other key variables were difficult to explain with the level of mean responses. For example, although attitudes showed similar levels of mean responses (i.e. $M=14.28$ versus 14.16 on a 21-point scale) for both groups, the direct effects of attitudes on behaviour were different across the two groups. Also, in the high effort group intentions scores were low (i.e. $M=7.96$ on a 14-point scale), but intentions had significant effects on behaviour. It should be noted here that the key paths are not affected by the means of the variables, because they are estimated on the basis of covariances. These findings suggest that the observed differences in the key paths cannot be fully accounted for in terms of the mean responses. Future research should investigate what mechanisms underlie the observed effects of the effort required for behaviour.

This study extends existing research on the attitude-behaviour relation in several ways. First, we examined a moderator of the causal processes of behavioural determination, providing a deeper understanding of the attitude-behaviour relation. Second, we investigated a behavioural quality (i.e. the effortfulness of behaviour) as a moderator of the attitude-behaviour relation. In contrast, past research on this issue has focused mainly on attitudinal qualities such as the consistency between affective and cognitive components of attitudes, the temporal stability of attitudes, confidence in attitudes, and the manner of attitude formation (e.g. Davidson and Jaccard, 1979; Fazio and Zanna, 1978, 1981). In addition to the qualities of attitudes and behaviour, the qualities of intentions might also be relevant. For example, the nature of intention formation has been found to have an impact on the attitude-behaviour relation: well-formed intentions may channel the effects of attitudes better than ill-formed intentions (Bagozzi and Yi, 1989).

A notable feature of this study is the measurement of behaviour. Previous studies have often used retrospective self-reports as indicators of behaviours, relying at times on single, dichotomous indicators of behaviour. The present study employed three measures of behaviour, including a more objective measure based on actual behaviour. Two measures were ratio scaled, the third interval.

In the way of caveats, the following is provided. First, this study was conducted with a single behaviour. Attempts to replicate the findings are needed in other behavioural domains. Second, other ways to operationalize the level of effort needed to perform a behaviour should be explored. Finally, factors related to effort needed such as involvement should be investigated. The present study examined a somewhat low involvement issue, but effort might function differently with highly involving acts or objects.

To the best of our knowledge, the present study is the first attempt to experimentally investigate conditions under which intentions do or do not mediate the effects of attitudes on behaviour (Bagozzi, Baumgartner and Yi, 1989). We found that intentions either function as mediators or they do not, depending on the level of effort needed for behaviour. An interesting theoretical question to address is whether attitudes can simultaneously affect behaviour in both direct and indirect (through intentions) senses. Several studies have documented such effects (e.g. Bentler and Speckart, 1979; Manstead et al., 1983; Zuckerman and Reis, 1978), but we are 
presently lacking a theory that can account for deliberate and less deliberate processes guiding behaviour. Is behaviour reasoned or unreasoned? Or can it be both? We have only begun to understand the role of intentions as mediators of the attitudebehaviour relation. The level of effort required to perform the behaviour represents one factor governing the role of intentions. But surely others remain to be found.

\section{REFERENCES}

Ajzen, I. and Fishbein, M. (1980). Understanding Attitudes and Predicting Social Behavior, Prentice-Hall, Englewood Cliffs.

Albrecht, S. L. and Carpenter, K. E. (1976). 'Attitudes as predictors of behaviors versus behavioral intentions: A convergence of research traditions', Sociometry, 39: 1-10.

Bagozzi, R. P., Baumgartner, J. and Yi, Y. (1989). 'An investigation into the role of intentions as mediators of the attitude-behavior relationship', Journal of Economic Psychology, 10: $35-62$.

Bagozzi, R. P. and Yi, Y. (1989). 'The degree of intention formation as a moderator of the attitude-behavior relation', Social Psychology Quarterly, 52.

Bem, D. J. (1972). 'Self-perception theory', In: Berkowitz, L. (Ed.) Advances in Experimental Social Psychology, Wiley, New York, pp. 1-62.

Bentler, P. M. and Speckart, G. (1979). 'Models of attitude-behavior relations', Psychological Review, 86: 452-464.

Bentler, P. M. and Speckart, G. (1981). 'Attitudes cause behaviors: A structural equation analysis', Journal of Personality and Social Psychology, 40: 226-238.

Davidson, A. R. and Jaccard, J. J. (1979). 'Variables that moderate the attitude-behavior relation: Results of a longitudinal study', Journal of Personality and Social Psychology, 37: 1364-1376.

Echabe, A. E., Rovira, D. P. and Garate, J. F. V. (1988). 'Testing Ajzen and Fishbein's attitudes models: the prediction of voting', European Journal of Social Psychology, 18: 181190.

Fazio, R. H. (1986). 'How do attitudes guide behavior?' In: Sorrentino, R. M. and Higgins, E. T. (Eds) Handbook of Motivation and Cognition: Foundations of Social Behavior, Guilford Press, New York, pp. 204-243.

Fazio, R. H. and Zanna, M. P. (1978). 'Attitudinal qualities relating to the strength of the attitude-behavior relationship', Journal of Experimental Social Psychology, 14: 398-408.

Fazio, R. H. and Zanna, M. P. (1981). 'Direct experience and attitude-behavior consistency', In: Berkowitz, L. (Ed.) Advances in Experimental Social Psychology, Academic Press, New York, pp. 161-202.

Festinger, L. (1957). A Theory of Cognitive Dissonance, Row, Evanston.

Fishbein, M. and Ajzen, I. (1975). Belief, Attitude, Intention and Behavior: An Introduction to Theory and Research, Addison-Wesley, Reading.

Fredricks, A. J. and Dossett, D. L. (1983). 'Attitude-behavior relations: A comparison of the Fishbein-Ajzen and the Bentler-Speckart models', Journal of Personality and Social Psychology, 45: 501-512.

Joreskog, K. G. (1971). 'Simultaneous factor analysis in several populations', Psychometrika, 36: $409-426$.

Joreskog, K. G. and Sorbom, D. (1984). LISREL VI: Analysis of Linear Structural Relationships by the Method of Maximum Likelihood, Scientific Software, Mooresville.

Kahle, L. R. and Berman, J. J. (1979). 'Attitudes cause behaviors: A cross-lagged panel analysis', Journal of Personality and Social Psychology, 37: 315-321.

Liska, A. E. (1984). 'A critical examination of the causal structure of the Fishbein-Ajzen model', Social Psychology Quarterly, 47: 61-74.

Manstead, A. S., Proffitt, C. and Smart, J. L. (1983). 'Predicting and understanding mothers' infant-feeding intention and behavior: Testing the theory of reasoned action', Journal of Personality and Social Psychology, 44: 657-671.

Roget's Thesaurus (1966). Dell Publishing, New York. 
Triandis, H. C. (1977). Interpersonal Behavior, Brooks. Cole, Monterey.

Warshaw, P. and Davis, F. (1985). 'Disentangling behavioral intention and behavioral expectation', Journal of Experimental Social Psychology, 21: 213-228.

Webster's New Collegiate Dictionary (1980). G \& C Merriam Company, Springfield.

Werts, C. E., Linn, R. L. and Joreskog, K. G. (1974). 'Intraclass reliability estimates: Testing structural assumptions', Educational and Psychological Measurement, 34: 25-33.

Zanna, M. P. and Fazio, R. H. (1982). 'The attitude-behavior relation: Moving toward a third generation of research', In: Zanna, M. P., Higgins, E. T. and Herman, C. P. (Eds) Consistency in Social Behavior: The Ontario Symposium, Vol. 2, Erlbaum, Hillsdale, pp. 283-301.

Zuckerman, M. and Reis, H. T. (1978). 'Comparison of three models for predicting altruistic behavior', Journal of Personality and Social Psychology, 36: 498-510. 